\title{
Erratum: Nuclear and neutron matter $G$-matrix calculations with a chiral effective field theory potential including effects of three-nucleon interactions [Phys. Rev. C 88, 064005 (2013)]
}

\author{
M. Kohno* \\ (Received 5 May 2017; revised manuscript received 31 July 2017; published 27 November 2017)
}

DOI: 10.1103/PhysRevC.96.059903

Several errors were found in the program code for the nuclear-matter calculation reported in the original paper. There also were errors in the spin-orbit and tensor terms of the effective two-nucleon forces derived from the three-nucleon forces (3NFs) of the chiral effective field theory (Ch-EFT). After correcting these errors, the enhancement of the tensor-force component is reduced to be about $15 \%$ from the previous value of about $30 \%$.

Although qualitatively important effects of the $3 \mathrm{NFs}$ for improving nuclear-matter saturation properties do not change, the saturation curve is modified quantitatively by the corrections. In the original paper, the low-energy constants $c_{D}=-4.381$ and $c_{E}=-1.126$ were used, referring to the nuclear-matter calculations with chiral low-momentum interactions by Hebeler et al. [1]. The results of the revised nuclear-matter calculation with the same $c_{D}$ 's and $c_{E}$ 's are shown in Figs. 2(a), 2(b), and 3(a).
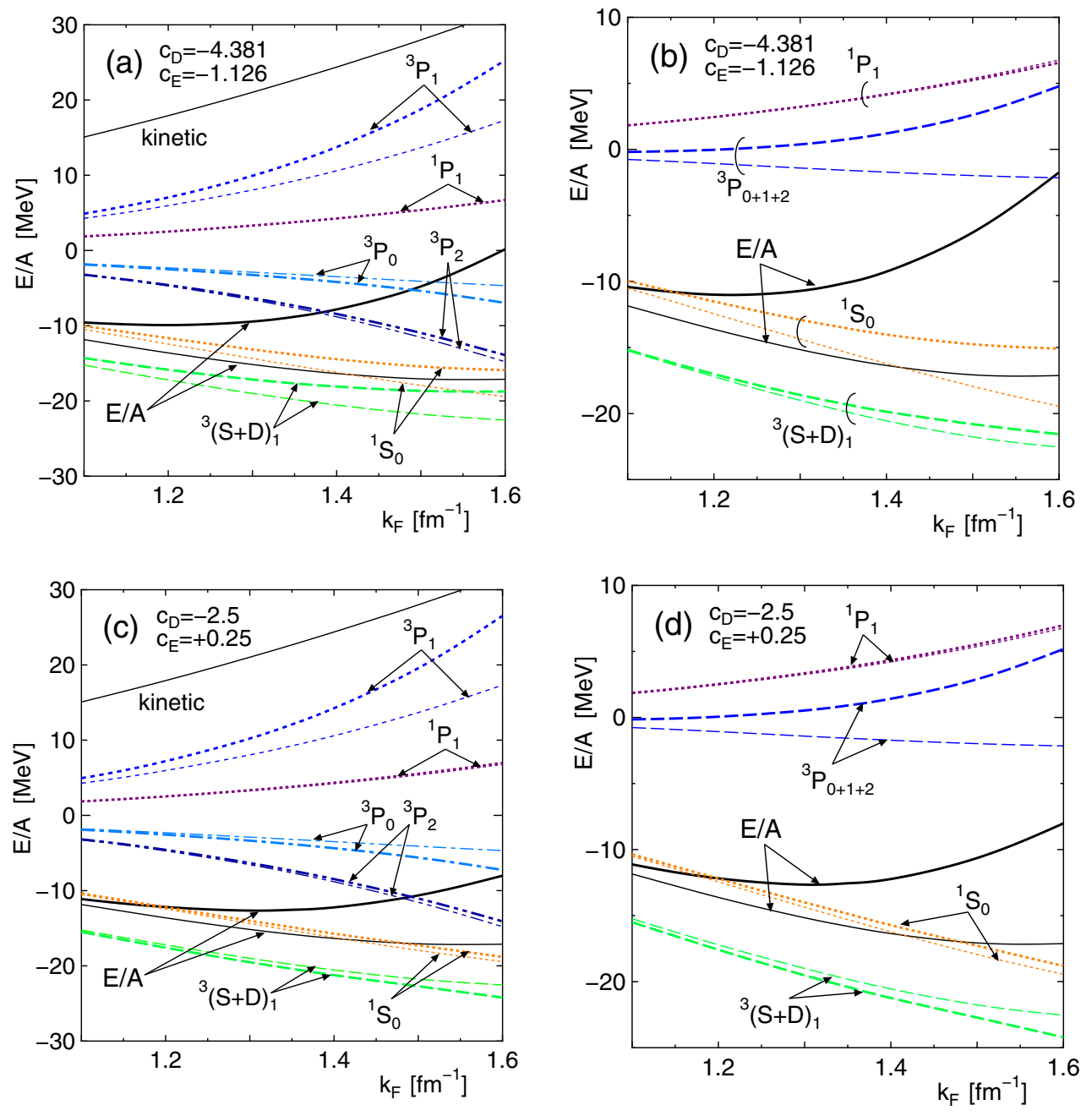

FIG. 2. Revised version of Fig. 2 in the original paper. $k_{F}$ dependence of partial-wave contributions to the nuclear-matter lowest-order Brueckner theory (LOBT) energy per nucleon for the Ch-EFT interaction for $\Lambda=550 \mathrm{MeV}$. The thick and thin curves are with and without the $3 \mathrm{NF}$ effects, respectively. The results with $\left(c_{D}, c_{E}\right)=(-4.381,-1.126)$ are shown in (a) full decomposition and (b) different $J$ 's being summed. The results with $\left(c_{D}, c_{E}\right)=(-2.5,0.25)$ are shown in (c) and (d). Note that the numerical value of the partial-wave contributions in Fig. 2 of the original paper was wrongly multiplied by $\frac{2}{3} k_{F}^{3}$. 

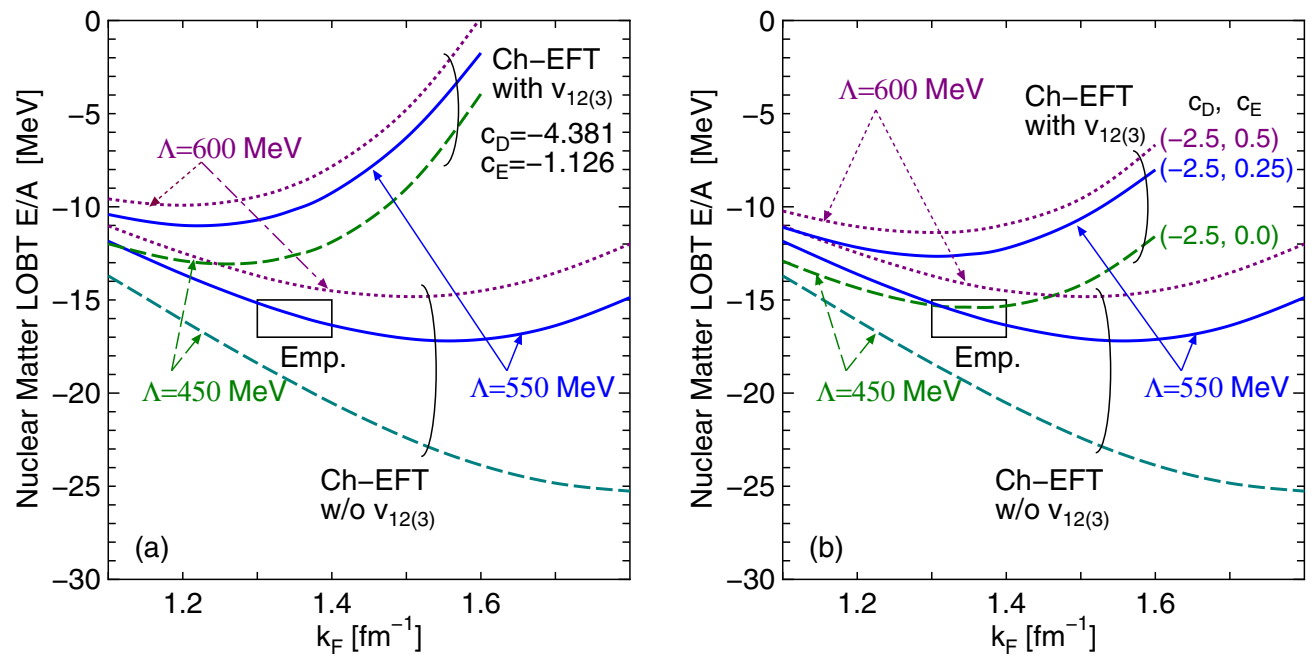

FIG. 3. Revised version of Fig. 3 in the original paper. LOBT energies per nucleon in symmetric nuclear matter for the Ch-EFT interaction with and without the $3 \mathrm{NF}$ effects. The left panel (a) represents the results with $\left(c_{D}, c_{E}\right)=(-4.381,-1.126)$. The right panel (b) shows the results with $c_{D}$ and $c_{E}$ refitted to obtain a better saturation curve.

To obtain a better saturation curve, the $c_{D}$ 's and $c_{E}$ 's have been refitted as shown in Fig. 3(b). The revised version of Fig. 2 in the original paper for partial-wave contributions is given as Figs. 2(c) and 2(d). Table II of the original paper for the spin-isospin decompositions is replaced by Table II in this Erratum. Because neutron-matter energies hardly change by the corrections, Fig. 6 of the original paper for the neutron-matter energy is not revised here.

The correction in the spin-orbit component changes the estimation of the strength of the one-body spin-orbit field in the form of the Scheerbaum factor, whereas the $c_{D}$ and $c_{E}$ terms do not contribute to the spin-orbit component. Table I in the original paper should be replaced by Table I, which indicates that the enhancement of the spin-orbit strength due to the $3 \mathrm{NF}$ is reduced to be about $20 \%$.

Changes in the $G$ matrices in nuclear matter including the $3 \mathrm{NF}$ effects affect the results of the subsequent applications of them to the description of the nucleon-nucleus and nucleus-nucleus scatterings reported in Refs. [2-4]. The corrected results are reported in separate Errata.

The author thanks S. Yoshida for finding errors in the program code and an omission of several terms in density-dependent effective two-body forces derived from the three-nucleon force of Ch-EFT.

\section{APPENDIX A}

The expression of the partial-wave expansion of the spin-orbit component of the $c_{3}$ term of $V_{C}$, Eq. (B14), should be corrected as

$$
\begin{aligned}
& \delta_{S 1} \frac{c_{3} g_{A}^{2}}{2 f_{\pi}^{4}} 3 \frac{\ell(\ell+1)+2-J(J+1)}{2 \ell+1}\left[\left(m_{\pi}^{2}+\frac{1}{2}\left(k_{1}^{\prime 2}+k_{1}^{2}\right)\right)\left\{Q_{W 0}^{\ell-1}\left(k_{1}^{\prime}, k_{1}\right)-Q_{W 0}^{\ell+1}\left(k_{1}^{\prime}, k_{1}\right)-W_{\ell s, 0}^{\ell}\left(k_{1}^{\prime}, k_{1}\right)\right\}\right. \\
& \left.-\delta_{\ell 1} \frac{k_{1}^{\prime} k_{1}}{2}\left[F_{0}\left(k_{1}^{\prime}\right)+F_{0}\left(k_{1}\right)-F_{1}\left(k_{1}^{\prime}\right)-F_{1}\left(K_{1}\right)\right]+k_{1}^{\prime} k_{1}\left\{\frac{\ell-1}{2 \ell-1} W_{\ell s, 0}^{\ell-1}\left(k_{1}^{\prime}, k_{1}\right)+\frac{\ell+2}{2 \ell+3} W_{\ell s, 0}^{\ell+1}\left(k_{1}^{\prime}, k_{1}\right)\right\}\right] .
\end{aligned}
$$

TABLE I. Revised version of Table I in the original paper. Scheerbaum factor $B_{S}$ is in units of $\mathrm{MeV} \mathrm{fm}^{5}$.

\begin{tabular}{lcccc}
\hline \hline & \multicolumn{2}{c}{ Nuclear matter } & \multicolumn{2}{c}{ Neutron matter } \\
\cline { 2 - 3 }$k_{F}=1.35 \mathrm{fm}^{-1}$ & $\mathrm{~N}^{3} \mathrm{LO}$ & $\mathrm{N}^{3} \mathrm{LO}+3 \mathrm{NF}$ & $\mathrm{N}^{3} \mathrm{LO}$ & $\mathrm{N}^{3} \mathrm{LO}+3 \mathrm{NF}$ \\
\hline$B_{S}(T=0)$ & 2.5 & -4.3 & 84.7 & 92.8 \\
$B_{S}(T=1)$ & 84.6 & 102.0 & $\mathrm{~N}^{3} \mathrm{LO}$ & $\mathrm{N}^{3} \mathrm{LO}+3 \mathrm{NF}$ \\
$k_{F}=1.07 \mathrm{fm}^{-1}$ & $\mathrm{~N}^{3} \mathrm{LO}$ & $\mathrm{N}^{3} \mathrm{LO}+3 \mathrm{NF}$ & -1.7 & 91.4 \\
$B_{S}(T=0)$ & 1.6 & 99.8 & 87.0 & 91.0 \\
$B_{S}(T=1)$ & 86.5 & &
\end{tabular}


TABLE II. Revised version of Table II in the original paper with $c_{D}$ and $c_{E}$ being tuned as in the text. Total energies and spin-isospin channel $\left({ }^{1} \mathrm{O},{ }^{3} \mathrm{E},{ }^{1} \mathrm{E}\right.$, and $\left.{ }^{3} \mathrm{O}\right)$ decompositions for three instances of the cutoff energy $\Lambda(\mathrm{MeV})$ at $k_{F}=1.07,1.35$, and $1.60 \mathrm{fm}^{-1}$ for symmetric nuclear matter and at $k_{F}=1.35$ and $1.80 \mathrm{fm}^{-1}$ for pure neutron matter. The corresponding density $\rho$ (fm ${ }^{-3}$ ), kinetic-energy $T$, and total potential-energy contribution $U$ also are tabulated. Energies are in units of $\mathrm{MeV}$.

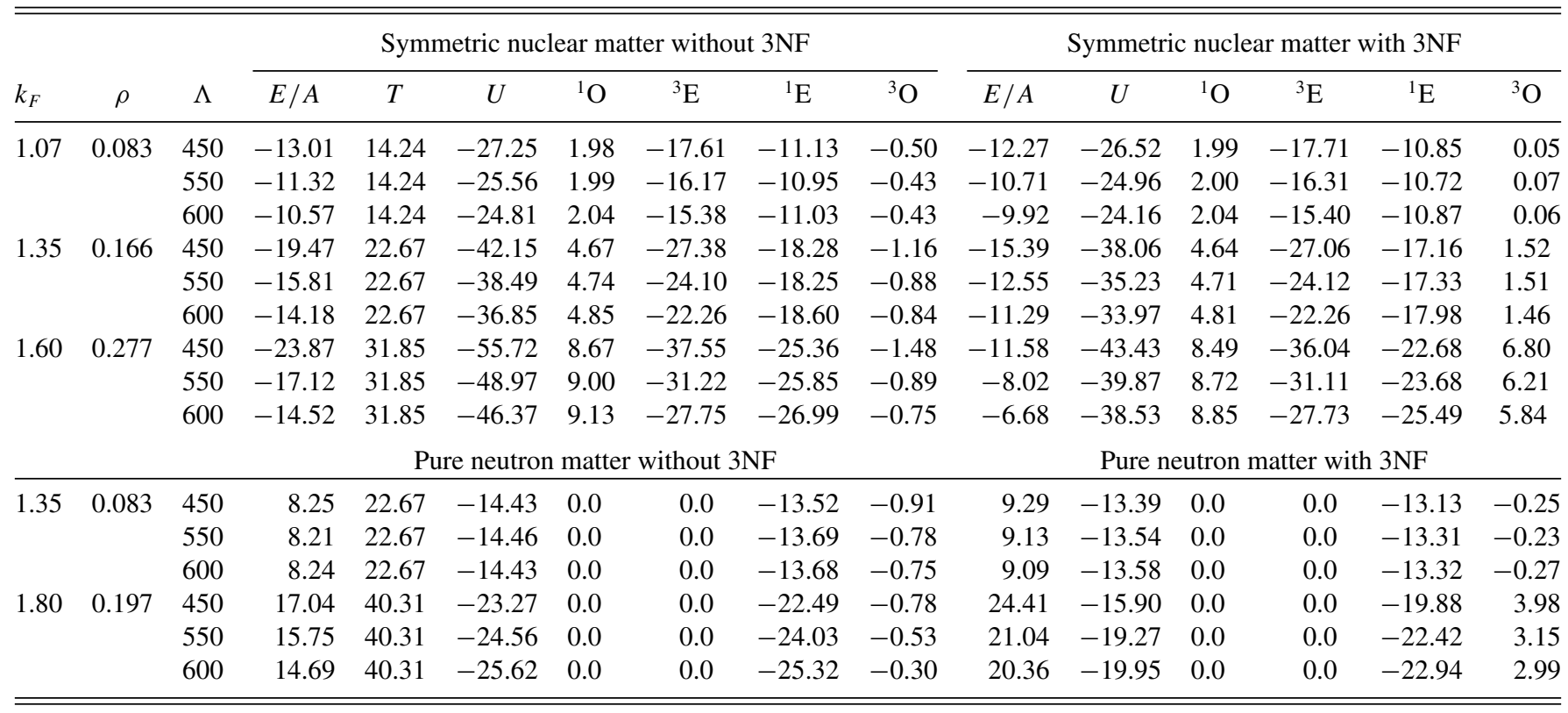

The spin-orbit component of the $c_{4}$ term of $V_{C}$, Eq. (A10), is absent in the original paper but present. The expression after the partial-wave expansion is

$$
\begin{aligned}
& \delta_{S 1} \frac{c_{4} g_{A}^{2}}{2 f_{\pi}^{4}}\left(\boldsymbol{\tau}_{1} \cdot \boldsymbol{\tau}_{2}\right) \frac{\ell(\ell+1)+2-J(J+1)}{2 \ell+1}\left[\left(m_{\pi}^{2}+\frac{1}{2}\left(k_{1}^{\prime 2}+k_{1}^{2}\right)\right)\left\{Q_{W 0}^{\ell-1}\left(k_{1}^{\prime}, k_{1}\right)-Q_{W 0}^{\ell+1}\left(k_{1}^{\prime}, k_{1}\right)-W_{\ell s, 0}^{\ell}\left(k_{1}^{\prime}, k_{1}\right)\right\}\right. \\
& \left.-\delta_{\ell 1} \frac{k_{1}^{\prime} k_{1}}{2}\left[F_{0}\left(k_{1}^{\prime}\right)+F_{0}\left(k_{1}\right)-F_{1}\left(k_{1}^{\prime}\right)-F_{1}\left(K_{1}\right)\right]+k_{1}^{\prime} k_{1}\left\{\frac{\ell-1}{2 \ell-1} W_{\ell s, 0}^{\ell-1}\left(k_{1}^{\prime}, k_{1}\right)+\frac{\ell+2}{2 \ell+3} W_{\ell s, 0}^{\ell+1}\left(k_{1}^{\prime}, k_{1}\right)\right\}\right] .
\end{aligned}
$$

\section{APPENDIX B}

In the original paper, tensor components in the form of $\left[\left[\sigma_{1} \times \sigma_{2}\right]^{2} \times\left[Y_{\ell}\left(\theta_{1}, \phi_{1}\right) \times Y_{\ell}\left(\theta_{2}, \phi_{2}\right)\right]^{2}\right]^{0}$ with $\ell \geqslant 2$ were ignored. Including them, the expression (B16) for the tensor components of the $c_{4}$ term of $V_{C}$, Eq. (A10) of the original paper, is replaced by

$$
\begin{aligned}
& 2 \frac{c_{4} g_{A}^{2}}{4 f_{\pi}^{4}} \frac{2}{3}\left(S_{12}\right)_{\ell 1 J}^{\ell^{\prime}}\left(\boldsymbol{\tau}_{1} \cdot \boldsymbol{\tau}_{2}\right)\left[\frac{1}{2 k_{1}^{\prime} k_{1}}\left[k_{1}^{\prime 2} Q_{\ell}(z)+k_{1}^{2} Q_{\ell^{\prime}}(z)-2 k_{1}^{\prime} k_{1} Q_{J}(z)\right]\left(\frac{1}{2} \rho_{0}-m_{\pi}^{2}\left[F_{0}\left(k_{1}^{\prime}\right)+F_{0}\left(k_{1}\right)\right]\right)\right. \\
& +\frac{k_{1}^{\prime} k_{1}}{2 J+1}\left[Q_{W 0}^{J+1}\left(k_{1}^{\prime}, k_{1}\right)-Q_{W 0}^{J-1}\left(k_{1}^{\prime}, k_{1}\right)\right] \\
& +\left[F_{0}\left(k_{1}^{\prime}\right)-2 F_{1}\left(k_{1}^{\prime}\right)\right]\left\{\frac{k_{1}^{\prime 2}}{4 k_{1}^{\prime} k_{1}}\left(-k_{1}^{\prime 2}+k_{1}^{2}+m_{\pi}^{2}\right) Q_{\ell}(z)-\frac{1}{4}\left(-k_{1}^{\prime 2}+k_{1}^{2}+m_{\pi}^{2}\right) Q_{J}(z)-\frac{1}{2} k_{1}^{\prime 2} \delta_{\ell 0}+\frac{1}{2} k_{1}^{\prime} k_{1} \delta_{J 0}\right\} \\
& +\left[F_{0}\left(k_{1}\right)-2 F_{1}\left(k_{1}\right)\right]\left\{\frac{k_{1}^{2}}{4 k_{1}^{\prime} k_{1}}\left(k_{1}^{\prime 2}-k_{1}^{2}+m_{\pi}^{2}\right) Q_{\ell^{\prime}}(z)-\frac{1}{4}\left(k_{1}^{\prime 2}-k_{1}^{2}+m_{\pi}^{2}\right) Q_{J}(z)-\frac{1}{2} k_{1}^{2} \delta_{\ell 0}+\frac{1}{2} k_{1}^{\prime} k_{1} \delta_{J 0}\right\} \\
& +\frac{1}{3} F_{3}\left(k_{1}^{\prime}\right)\left\{-\frac{3}{2} k_{1}^{\prime 2} \delta_{\ell 0}+\frac{3}{2} k_{1}^{\prime} k_{1} \delta_{J 0}+\frac{k_{1}^{\prime 2}}{4 k_{1}^{\prime} k_{1}}\left(-k_{1}^{\prime 2}+3 k_{1}^{2}+3 m_{\pi}^{2}\right) Q_{\ell}(z)-\frac{1}{4}\left(k_{1}^{\prime 2}+3 k_{1}^{2}+3 m_{\pi}^{2}\right) Q_{J}(z)+\frac{1}{2} k_{1}^{\prime} k_{1} Q_{\ell^{\prime}}(z)\right\} \\
& +\frac{1}{3} F_{3}\left(k_{1}\right)\left\{-\frac{3}{2} k_{1}^{2} \delta_{\ell 0}+\frac{3}{2} k_{1}^{\prime} k_{1} \delta_{J 0}+\frac{k_{1}^{2}}{4 k_{1}^{\prime} k_{1}}\left(-k_{1}^{2}+3 k_{1}^{\prime 2}+3 m_{\pi}^{2}\right) Q_{\ell^{\prime}}(z)-\frac{1}{4}\left(k_{1}^{2}+3 k_{1}^{\prime 2}+3 m_{\pi}^{2}\right) Q_{J}(z)+\frac{1}{2} k_{1}^{\prime} k_{1} Q_{\ell}(z)\right\} \\
& \left.-\frac{1}{3} F_{2}\left(k_{1}^{\prime}\right) \frac{k_{1}^{\prime 2}}{2 k_{1}^{\prime} k_{1}}\left\{k_{1}^{\prime 2} Q_{\ell}(z)+k_{1}^{2} Q_{\ell^{\prime}}(z)-2 k_{1}^{\prime} k_{1} Q_{J}(z)\right\}-\frac{1}{3} F_{2}\left(k_{1}\right) \frac{k_{1}^{2}}{2 k_{1}^{\prime} k_{1}}\left\{k_{1}^{2} Q_{\ell^{\prime}}(z)+k_{1}^{\prime 2} Q_{\ell}(z)-2 k_{1}^{\prime} k_{1} Q_{J}(z)\right\}\right] \\
& +\sqrt{\frac{5}{6}} \frac{(-1)^{J}}{\left(\ell^{\prime}, 2, \ell\right)}\left(k_{1}^{\prime 2}+k_{1}^{2}\right)\left\{Q_{W 1}^{\ell^{\prime} J}\left(k_{1}^{\prime}, k_{1}\right)+Q_{W 1}^{\ell J}\left(k_{1}, k_{1}^{\prime}\right)\right\}\left(\ell^{\prime} 010 \mid J 0\right)(\ell 010 \mid J 0)\left\{\begin{array}{ccc}
1 & 2 & 1 \\
\ell^{\prime} & J & \ell
\end{array}\right\}
\end{aligned}
$$


$+\frac{\sqrt{2}}{3} \frac{(-1)^{J+1}}{\left(\ell^{\prime}, 2, \ell\right)} \sum_{j^{\prime} j k} k_{1}^{\prime} k_{1}\left[Q_{W 1}^{j^{\prime} k}\left(k_{1}^{\prime}, k_{1}\right)+Q_{W 1}^{j k}\left(k_{1}, k_{1}^{\prime}\right)\right] \hat{k}\left(10 k 0 \mid j^{\prime} 0\right)(10 k 0 \mid j 0)\left\{\begin{array}{lll}1 & 2 & 1 \\ j^{\prime} & k & j\end{array}\right\}\left\{\begin{array}{lll}j^{\prime} & j & 2 \\ \ell & \ell^{\prime} & 1\end{array}\right\}\left(10 \ell^{\prime} 0 \mid j^{\prime} 0\right)(10 \ell 0 \mid j 0)$ $+\frac{1}{3}\left\{k_{1}^{\prime 2}\left\{Q_{X 1}^{\ell}\left(k_{1}, k_{1}^{\prime}\right)+Q_{X 1}^{\ell}\left(k_{1}^{\prime}, k_{1}\right)-\delta_{\ell 0} \frac{1}{2}\left[F_{0}\left(k_{1}^{\prime}\right)+F_{0}\left(k_{1}\right)\right]\right\}+k_{1}^{2}\left(Q_{X 1}^{\ell^{\prime}}\left\{k_{1}, k_{1}^{\prime}\right)+Q_{X 1}^{\ell^{\prime}}\left(k_{1}^{\prime}, k_{1}\right)-\delta_{\ell^{\prime} 0} \frac{1}{2}\left[F_{0}\left(k_{1}^{\prime}\right)+F_{0}\left(k_{1}\right)\right]\right\}\right.$ $\left.-2 k_{1}^{\prime} k_{1}\left\{Q_{X 1}^{J}\left(k_{1}, k_{1}^{\prime}\right)+Q_{X 1}^{J}\left(k_{1}^{\prime}, k_{1}\right)-\delta_{J 0} \frac{1}{2}\left[F_{0}\left(k_{1}^{\prime}\right)+F_{0}\left(k_{1}\right)\right]\right\}\right\}$

$+\sqrt{\frac{35}{3}} \frac{(-1)^{J}}{\left(\ell^{\prime}, 2, \ell\right)} \sum_{j k} k_{1}^{\prime 2}\left[Q_{W 1}^{j k}\left(k_{1}^{\prime}, k_{1}\right)+Q_{W 1}^{\ell k}\left(k_{1}, k_{1}^{\prime}\right)\right] \hat{j}(j 010 \mid k 0)(10 \ell 0 \mid k 0)\left\{\begin{array}{lll}1 & 2 & 1 \\ j & k & \ell\end{array}\right\}\left\{\begin{array}{lll}2 & j & \ell^{\prime} \\ \ell & 2 & 2\end{array}\right\}\left(20 j 0 \mid \ell^{\prime} 0\right)$ $+\sqrt{\frac{35}{3}} \frac{(-1)^{J}}{\left(\ell^{\prime}, 2, \ell\right)} \sum_{j k} k_{1}^{2}\left[Q_{W 1}^{j k}\left(k_{1}, k_{1}^{\prime}\right)+Q_{W 1}^{\ell^{\prime} k}\left(k_{1}^{\prime}, k_{1}\right)\right] \hat{j}(j 010 \mid k 0)\left(10 \ell^{\prime} 0 \mid k 0\right)\left\{\begin{array}{lll}1 & 2 & 1 \\ j & k & \ell^{\prime}\end{array}\right\}\left\{\begin{array}{lll}2 & j & \ell \\ \ell^{\prime} & 2 & 2\end{array}\right\}(20 j 0 \| \mid \ell 0)$ $+\sqrt{\frac{35}{2}} \frac{(-1)^{J}}{\left(\ell^{\prime}, 2, \ell\right)} \sum_{j^{\prime} j k} \hat{j}^{\prime} \hat{j}\left(10 j^{\prime} 0 \mid k 0\right)(10 j 0 \mid k 0)\left\{\begin{array}{ccc}1 & 2 & 1 \\ j^{\prime} & k & j\end{array}\right\}\left\{\begin{array}{lll}1 & j^{\prime} & \ell^{\prime} \\ 1 & j & \ell \\ 2 & 2 & 2\end{array}\right\}\left(10 j^{\prime} 0 \mid \ell^{\prime} 0\right)(10 j 0 \mid \ell 0) 2 k_{1}^{\prime} k_{1}\left[Q_{W 1}^{j^{\prime} k}\left(k_{1}^{\prime}, k_{1}\right)+Q_{W 1}^{j^{\prime} j}\left(k_{1}, k_{1}^{\prime}\right)\right]$ $-\frac{2}{3}\left\{k_{1}^{\prime 2} Q_{W 2}^{\ell}\left(k_{1}^{\prime}, k_{1}\right)+k_{1}^{2} Q_{W 2}^{\ell^{\prime}}\left(k_{1}^{\prime}, k_{1}\right)-2 k_{1}^{\prime} k_{1} Q_{W 2}^{J}\left(k_{1}^{\prime}, k_{1}\right)\right\}-\frac{1}{3 \sqrt{5}} \frac{1}{\left(\ell^{\prime}, 2, \ell\right)} Q_{W 2}^{\ell^{\prime} \ell}\left(k_{1}^{\prime}, k_{1}\right)\left(\ell^{\prime} 0 \ell 0 \mid 20\right)\left(k_{1}^{\prime 2}+k_{1}^{2}\right)$ $-\frac{1}{15 \sqrt{3 \hat{\ell}^{\prime} \ell}} \frac{1}{\left(\ell^{\prime}, 2, \ell\right)} \sum_{j^{\prime} j} \hat{j^{\prime}} \hat{j} Q_{W 2}^{j^{\prime} j}\left(k_{1}^{\prime}, k_{1}\right) \frac{1}{5}\left(j^{\prime} 0 j 0 \mid 20\right) 2 k_{1}^{\prime} k_{1}\left\{\begin{array}{ccc}j^{\prime} & j & 2 \\ \ell & \ell^{\prime} & 1\end{array}\right\}\left(10 j^{\prime} 0 \mid \ell^{\prime} 0\right)(10 j 0 \mid \ell 0)$ $+\frac{\sqrt{14}}{3} \frac{(-1)^{\ell+1}}{\left(\ell^{\prime}, 2, \ell\right)} \sum_{j}\left(\ell^{\prime} 020 \mid j 0\right)(\ell 020 \mid j 0)\left\{\begin{array}{lll}\ell^{\prime} & \ell & 2 \\ 2 & 2 & j\end{array}\right\}\left\{\sqrt{\hat{\ell}^{\prime}} k_{1}^{\prime 2} Q_{W 2}^{j^{\prime} \ell}\left(k_{1}^{\prime}, k_{1}\right)+\sqrt{\hat{\ell}} k_{1}^{2} Q_{W 2}^{\ell^{\prime} j}\left(k_{1}^{\prime}, k_{1}\right)\right\}$ $+\sqrt{\frac{7}{15}} \frac{1}{\left(\ell^{\prime}, 2, \ell\right)} \sum_{j^{\prime} j} \hat{j}^{\prime} \hat{j} 2 k_{1}^{\prime} k_{1} Q_{W 2}^{j^{\prime} j}\left(k_{1}^{\prime}, k_{1}\right)\left(j^{\prime} 0 j 0 \mid 20\right)\left\{\begin{array}{ccc}1 & j^{\prime} & \ell^{\prime} \\ 1 & j & \ell \\ 2 & 2 & 2\end{array}\right\}\left(10 j^{\prime} 0 \mid \ell^{\prime} 0\right)(10 j 0 \mid \ell 0)$ for $\ell^{\prime}=\ell \pm 2(J=\ell \pm 1)$ and

$$
\begin{aligned}
& 2 \frac{c_{4} g_{A}^{2}}{4 f_{\pi}^{4}} \frac{2}{3}\left(S_{12}\right)_{\ell 1 J}^{\ell^{\prime}}\left(\boldsymbol{\tau}_{1} \cdot \boldsymbol{\tau}_{2}\right)\left[\left(\frac{1}{2} \rho_{0}-m_{\pi}^{2}\left[F_{0}\left(k_{1}^{\prime}\right)+F_{0}\left(k_{1}\right)\right]\right)\left\{\frac{k_{1}^{2}+k_{1}^{2}}{2 k_{1}^{\prime} k_{1}} Q_{\ell}(z)-\frac{1}{2} \frac{2 \ell+3}{2 \ell+1} Q_{\ell-1}(z)-\frac{1}{2} \frac{2 \ell-1}{2 \ell+1} Q_{\ell+1}(z)\right\}\right. \\
& \quad+k_{1}^{\prime} k_{1}\left\{\left(\frac{(2 \ell+1)^{2}}{(2 \ell-1)(2 \ell+3)}-2\right) Q_{W 0}^{\ell}\left(k_{1}^{\prime}, k_{1}\right)+\frac{(2 \ell+3)(\ell-1)}{(2 \ell+1)(2 \ell-1)} Q_{W 0}^{\ell-2}\left(k_{1}^{\prime}, k_{1}\right)+\frac{(2 \ell-1)(\ell+1)}{(2 \ell+1)(2 \ell+3)} Q_{W 0}^{\ell+2}\left(k_{1}^{\prime}, k_{1}\right)\right\} \\
& \quad+\left[F_{0}\left(k_{1}^{\prime}\right)-2 F_{1}\left(k_{1}^{\prime}\right)\right]\left\{-\frac{1}{2} k_{1}^{\prime 2} \delta_{\ell 0}+\frac{5}{12} k_{1}^{\prime} k_{1} \delta_{\ell 1}+\frac{k_{1}^{\prime 2}}{4 k_{1}^{\prime} k_{1}}\left(-k_{1}^{\prime 2}+k_{1}^{2}+m_{\pi}^{2}\right) Q_{\ell}(z)\right. \\
& \left.\quad-\frac{1}{8(2 \ell+1)}\left(-k_{1}^{\prime 2}+k_{1}^{2}+m_{\pi}^{2}\right)\left[(2 \ell+3) Q_{\ell-1}(z)+(2 \ell-1) Q_{\ell+1}(z)\right]\right\} \\
& \quad+\frac{1}{3} F_{3}\left(k_{1}^{\prime}\right)\left\{-\frac{3}{2} k_{1}^{\prime 2} \delta_{\ell 0}+\frac{5}{4} k_{1}^{\prime} k_{1} \delta_{\ell 1}+\frac{k_{1}^{\prime 2}}{4 k_{1}^{\prime} k_{1}}\left[\left(-k_{1}^{\prime 2}+5 k_{1}^{2}+3 m_{\pi}^{2}\right) Q_{\ell}(z)\right]\right. \\
& \left.\quad-\frac{1}{8(2 \ell+1)}\left(k_{1}^{\prime 2}+3 k_{1}^{2}+3 m_{\pi}^{2}\right)\left[(2 \ell+3) Q_{\ell-1}(z)+(2 \ell-1) Q_{\ell+1}(z)\right]\right\} \\
& +\left[F_{0}\left(k_{1}\right)-2 F_{1}\left(k_{1}\right)\right]\left\{-\frac{1}{2} k_{1}^{2} \delta_{\ell 0}+\frac{5}{12} k_{1}^{\prime} k_{1} \delta_{\ell 1}+\frac{k_{1}^{2}}{4 k_{1}^{\prime} k_{1}}\left(k_{1}^{\prime 2}-k_{1}^{2}+m_{\pi}^{2}\right) Q_{\ell}(z)\right. \\
& \left.\quad-\frac{1}{8(2 \ell+1)}\left(k_{1}^{\prime 2}-k_{1}^{2}+m_{\pi}^{2}\right)\left[(2 \ell+3) Q_{\ell-1}(z)+(2 \ell-1) Q_{\ell+1}(z)\right]\right\}+\frac{1}{3} F_{3}\left(k_{1}\right)\left\{-\frac{3}{2} k_{1}^{2} \delta_{\ell 0}+\frac{5}{4} k_{1}^{\prime} k_{1} \delta_{\ell 1}\right. \\
& \left.+\frac{k_{1}^{2}}{4 k_{1}^{\prime} k_{1}}\left(-k_{1}^{2}+5 k_{1}^{\prime 2}+3 m_{\pi}^{2}\right) Q_{\ell}(z)-\frac{1}{8(2 \ell+1)}\left(k_{1}^{2}+3 k_{1}^{\prime 2}+3 m_{\pi}^{2}\right)\left[(2 \ell+3) Q_{\ell-1}(z)+(2 \ell-1) Q_{\ell+1}(z)\right]\right\}
\end{aligned}
$$




$$
\begin{aligned}
& -\frac{1}{3}\left[k_{1}^{\prime 2} F_{2}\left(k_{1}^{\prime}\right)+k_{1}^{2} F_{2}\left(k_{1}\right)\right]\left\{\frac{k_{1}^{\prime 2}+k_{1}^{2}}{2 k_{1}^{\prime} k_{1}} Q_{\ell}(z)-\frac{1}{\hat{\ell}}\left[(2 \ell+3) Q_{\ell-1}(z)+(2 \ell-1) Q_{\ell+1}(z)\right]\right\} \\
& +\frac{1}{3}\left(k_{1}^{\prime 2}+k_{1}^{2}\right)\left\{\frac{(2 \ell+3)}{2 \hat{\ell}}\left[Q_{W 1}^{\ell \ell-1}\left(k_{1}^{\prime}, k_{1}\right)+Q_{W 1}^{\ell \ell-1}\left(k_{1}, k_{1}^{\prime}\right)\right]+\frac{(2 \ell-1)}{2 \hat{\ell}}\left[Q_{W 1}^{\ell \ell+1}\left(k_{1}^{\prime}, k_{1}\right)+Q_{W 1}^{\ell \ell+1}\left(k_{1}, k_{1}^{\prime}\right)\right]\right\} \\
& +\frac{\sqrt{2}}{3} \frac{(-1)^{\ell}}{(\ell, 2, \ell)} \sum_{j^{\prime} j k} k_{1}^{\prime} k_{1}\left\{Q_{W 1}^{j^{\prime} k}\left(k_{1}^{\prime}, k_{1}\right)+Q_{W 1}^{j^{\prime} k}\left(k_{1}, k_{1}^{\prime}\right)\right\} \sqrt{\hat{j^{\prime}} \hat{j}}\left(10 j^{\prime} 0 \mid k 0\right)(10 j 0 \mid k 0)\left\{\begin{array}{lll}
1 & 2 & 1 \\
j^{\prime} & k & j
\end{array}\right\}\left\{\begin{array}{lll}
j^{\prime} & j & 2 \\
\ell & \ell & 1
\end{array}\right\}\left(10 \ell 0 \mid j^{\prime} 0\right)(10 \ell 0 \mid j 0) \\
& +\frac{1}{3}\left\{\left(k_{1}^{\prime 2}+k_{1}^{2}\right)\left(Q_{X 1}^{\ell}\left(k_{1}, k_{1}^{\prime}\right)+Q_{X 1}^{\ell}\left(k_{1}^{\prime}, k_{1}\right)-\delta_{\ell 0} \frac{1}{2}\left[F_{0}\left(k_{1}^{\prime}\right)+F_{0}\left(k_{1}\right)\right]\right)+\frac{5}{6} k_{1}^{\prime} k_{1} \delta_{\ell 1}\left[F_{0}\left(k_{1}^{\prime}\right)+F_{0}\left(k_{1}\right)\right]\right. \\
& \left.-2 k_{1}^{\prime} k_{1} \frac{1}{2 \hat{\ell}}\left\{(2 \ell+3)\left[Q_{X 1}^{\ell-1}\left(k_{1}, k_{1}^{\prime}\right)+Q_{X 1}^{\ell-1}\left(k_{1}^{\prime}, k_{1}\right)\right]+(2 \ell-1)\left[Q_{X 1}^{\ell+1}\left(k_{1}, k_{1}^{\prime}\right)+Q_{X 1}^{\ell+1}\left(k_{1}^{\prime}, k_{1}\right)\right]\right\}\right\} \\
& +\sqrt{\frac{35}{3}} \frac{1}{(\ell, 2, \ell)} \sum_{j^{\prime} j}\left\{k_{1}^{\prime 2} Q_{W 1}^{j^{\prime} j}\left(k_{1}^{\prime}, k_{1}\right)+k_{1}^{2} Q_{W 1}^{j^{\prime} j}\left(k_{1}, k_{1}^{\prime}\right)\right\} \hat{j}^{\prime}(-1)^{j}\left(j^{\prime} 010 \mid j 0\right)(10 \ell 0 \mid j 0)\left\{\begin{array}{lll}
1 & 2 & 1 \\
j^{\prime} & j & \ell
\end{array}\right\}\left\{\begin{array}{lll}
2 & j^{\prime} & \ell \\
\ell & 2 & 2
\end{array}\right\}\left(20 j^{\prime} 0 \mid \ell 0\right) \\
& +\sqrt{\frac{35}{3}} \frac{1}{(\ell, 2, \ell)} \sum_{j^{\prime} j}\left\{k_{1}^{2} Q_{W 1}^{\ell j}\left(k_{1}^{\prime}, k_{1}\right)+k_{1}^{\prime 2} Q_{W 1}^{\ell j}\left(k_{1}, k_{1}^{\prime}\right)\right\} \hat{j}^{\prime}(-1)^{j}(\ell 010 \mid j 0)\left(10 j^{\prime} 0 \mid j 0\right)\left\{\begin{array}{lll}
1 & 2 & 1 \\
\ell & j & j^{\prime}
\end{array}\right\}\left\{\begin{array}{lll}
2 & j^{\prime} & \ell \\
\ell & 2 & 2
\end{array}\right\}\left(20 j^{\prime} 0 \mid \ell 0\right) \\
& -\sqrt{\frac{35}{2}} \frac{(-1)^{\ell}}{(\ell, 2, \ell)} \sum_{j^{\prime} j k} 2 k_{1}^{\prime} k_{1}\left\{Q_{W 1}^{j^{\prime} k}\left(k_{1}^{\prime}, k_{1}\right)+Q_{W 1}^{j^{\prime} k}\left(k_{1}, k_{1}^{\prime}\right)\right\} \hat{j}^{\prime} \hat{j}\left(10 j^{\prime} 0 \mid k 0\right)(10 j 0 \mid k 0)\left\{\begin{array}{lll}
1 & 2 & 1 \\
j^{\prime} & k & j
\end{array}\right\}\left\{\begin{array}{lll}
1 & j^{\prime} & \ell \\
1 & j & \ell \\
2 & 2 & 2
\end{array}\right\}\left(10 j^{\prime} 0 \mid \ell 0\right)(10 j 0 \mid \ell 0) \\
& -\frac{1}{3}\left\{2\left(k_{1}^{\prime 2}+k_{1}^{2}\right) Q_{W 2}^{\ell}\left(k_{1}^{\prime}, k_{1}\right)-2 k_{1}^{\prime} k_{1} \frac{1}{2 \hat{\ell}}\left\{(2 \ell+3) Q_{W 2}^{\ell-1}\left(k_{1}^{\prime}, k_{1}\right)+(2 \ell-1) Q_{W 2}^{\ell+1}\left(k_{1}^{\prime}, k_{1}\right)\right\}\right\} \\
& -\frac{1}{15 \sqrt{3}} \frac{1}{(\ell, 2, \ell)} \sum_{j^{\prime} j} 2 k_{1}^{\prime} k_{1} Q_{W 2}^{j^{\prime} j}\left(k_{1}^{\prime}, k_{1}\right) \sqrt{\hat{j}^{\prime} \hat{j}}\left(j^{\prime} 0 j 0 \mid 20\right)\left\{\begin{array}{lll}
j^{\prime} & j & 2 \\
\ell & \ell & 1
\end{array}\right\}\left(10 \ell 0 \mid j^{\prime} 0\right)(10 \ell 0 \mid j 0) \\
& -\frac{1}{3} \sqrt{\frac{14}{5}} \frac{1}{(\ell, 2, \ell)} \sum_{j}\left\{k_{1}^{\prime 2} Q_{W 2}^{j \ell}\left(k_{1}^{\prime}, k_{1}\right)+k_{1}^{2} Q_{W 2}^{\ell j}\left(k_{1}^{\prime}, k_{1}\right)\right\} \hat{j}(j 0 \ell 0 \mid 20)\left\{\begin{array}{lll}
2 & j & \ell \\
\ell & 2 & 2
\end{array}\right\}(20 j 0 \mid \ell 0) \\
& \left.+\sqrt{\frac{7}{15}} \frac{1}{(\ell, 2, \ell)} \sum_{j^{\prime} j} 2 k_{1}^{\prime} k_{1} Q_{W 2}^{j^{\prime} j}\left(k_{1}^{\prime}, k_{1}\right) \hat{j^{\prime}} \hat{j}\left(j^{\prime} 0 j 0 \mid 20\right)\left\{\begin{array}{ccc}
1 & j^{\prime} & \ell \\
1 & j & \ell \\
2 & 2 & 2
\end{array}\right\}\left(10 j^{\prime} 0 \mid \ell 0\right)(10 j 0 \mid \ell 0)\right]
\end{aligned}
$$

for $\ell^{\prime}=\ell=J \pm 1$. The notation $\left(j_{1}, j_{2}, j_{3}\right)$ stands for an abbreviation of a 3-J symbol of $\left(\begin{array}{ccc}j_{1} & j_{2} & j_{3} \\ 0 & 0 & 0\end{array}\right) . \hat{\ell}$ and $z$ mean $\hat{\ell} \equiv 2 \ell+1$ and $z \equiv \frac{k_{1}^{\prime 2}+k_{1}^{2}+m_{\pi}^{2}}{2 k_{1}^{\prime} k_{1}}$. Definitions of $F_{0}, F_{1}, Q_{W 1}^{\ell}, Q_{W 2}^{\ell}$, and $W_{\ell s, 0}$ are found in the original paper as Eqs. (A13), (A14), (B1), (B2), and (B10), respectively. $Q_{W 1}^{\ell^{\prime} \ell}, Q_{W 2}^{\ell^{\prime} \ell}$, and $Q_{X 1}^{\ell}$ represent the following integrals involving a second kind of Legendre function $Q_{\ell}$ :

$$
\begin{aligned}
& Q_{W 1}^{\ell^{\prime} \ell}\left(k_{1}^{\prime}, k_{1}\right) \equiv \frac{1}{(2 \pi)^{2}} \frac{1}{2 k_{1}^{\prime}} \int_{0}^{k_{F}} d k_{3} k_{3} Q_{\ell^{\prime}}\left(x^{\prime}\right) Q_{\ell}(x), \\
& Q_{W 2}^{\ell^{\prime} \ell}\left(k_{1}^{\prime}, k_{1}\right) \equiv \frac{1}{(2 \pi)^{2}} \frac{1}{2 k_{1}^{\prime} k_{1}} \int_{0}^{k_{F}} d k_{3} k_{3}^{2} Q_{\ell^{\prime}}\left(x^{\prime}\right) Q_{\ell}(x), \\
& Q_{X 1}^{\ell}\left(k_{1}^{\prime}, k_{1}\right) \equiv \frac{\ell}{2 \ell+1} Q_{W 1}^{\ell \ell-1}\left(k_{1}, k_{1}^{\prime}\right)+\frac{\ell+1}{2 \ell+1} Q_{W 1}^{\ell \ell+1}\left(k_{1}, k_{1}^{\prime}\right),
\end{aligned}
$$

where $x^{\prime} \equiv \frac{k_{1}^{2}+k_{3}^{2}+m_{\pi}^{2}}{2 k_{1}^{\prime} k_{3}}$ and $x \equiv \frac{k_{1}^{2}+k_{3}^{2}+m_{\pi}^{2}}{2 k_{1} k_{3}} . Q_{W 1}^{\ell \ell}\left(k_{1}^{\prime}, k_{1}\right)$ is equal to $Q_{W 1}^{\ell}\left(k_{1}^{\prime}, k_{1}\right)$ defined by Eq. (B5) in the original paper.

[1] K. Hebeler, S. K. Bogner, R. J. Furnstahl, A. Nogga, and A. Schwenk, Phys. Rev. C 83, 031301(R) (2011).

[2] K. Minomo, M. Toyokawa, M. Kohno, and M. Yahiro, Phys. Rev. C 90, 051601 (2014).

[3] M. Toyokawa, M. Yahiro, T. Matsumoto, K. Minomo, K. Ogata, and M. Kohno, Phys. Rev. C 92, 024618 (2015).

[4] K. Minomo, M. Kohno, and K. Ogata, Phys. Rev. C 93, 014607 (2016). 